\title{
Progranulin protects the mouse retina under hypoxic conditions via inhibition of the Toll-like receptor-4-NADPH oxidase 4 signaling pathway
}

\author{
ZHI-PENG YOU*, MENG-JIA YU*, YU-LAN ZHANG and KE SHI
}

Department of Ophthalmology, The Second Affiliated Hospital of Nanchang University, Nanchang, Jiangxi 330006, P.R. China

Received December 14, 2017; Accepted October 12, 2018

DOI: $10.3892 / \mathrm{mmr} .2018 .9634$

\begin{abstract}
To investigate the function of progranulin on the retina under hypoxic conditions, 8-week-old C57BL/6 mice were divided into normal condition and hypoxic condition groups ( $\mathrm{n}=24 \mathrm{mice} / \mathrm{group})$. The hypoxia model was established through intravitreal injection of $9 \mathrm{mM}$ cobalt chloride. Subsequently, $10 \mathrm{mM}$ progranulin and an equal amount of PBS were injected into the right and left eyes, respectively. Photoreceptor function was examined using electroretinogram (ERG) analysis. Morphological alterations were examined using immunofluorescence co-localization, retinal vascular inflammation was examined using the leukostasis assay, and signaling pathways were screened using immunoblotting. The results revealed that ERG amplitude was significantly lower under hypoxic conditions compared with under normal conditions. Furthermore, the amplitude was significantly reduced in the PBS-injected eyes compared with in the progranulin-injected eyes. Morphological examination demonstrated that the number of rods in the PBS-injected eyes was decreased compared with in the progranulin-injected eyes under hypoxic conditions. In addition, the arrangement of the cones was sparse and the morphology of the outer segments was short and small. Although the number of adherent leukocytes in the progranulin-injected eyes was higher in the hypoxic mice compared with in those under normal conditions, the number was only $52.31 \%$ of the number detected in the PBS-injected eyes. Analysis of the signaling pathways demonstrated that the protective effects of progranulin on retinas under hypoxic conditions were regulated by the
\end{abstract}

Correspondence to: Dr Ke Shi, Department of Ophthalmology, The Second Affiliated Hospital of Nanchang University, 1 Minde Road, Nanchang, Jiangxi 330006, P.R. China

E-mail: 86325294@qq.com

${ }^{*}$ Contributed equally

Abbreviations: TLR4, Toll-like receptor 4; NOX4, NADPH oxidase 4; TNF, tumor necrosis factor; GSK, glycogen synthase kinase; PNA, peanut agglutinin

Key words: progranulin, hypoxia, retina, TLR4-NOX4 signaling pathway
Toll-like receptor 4 (TLR4)-NADPH oxidase 4 (NOX4) pathway, instead of the caspase and $\mathrm{Wnt} / \beta$-catenin pathways. In conclusion, progranulin exerted protective effects on the function and morphology of photoreceptors in a hypoxic environment, and could reduce retinal vascular inflammation, through inhibition of the TLR4-NOX4 pathway.

\section{Introduction}

Hypoxia is an important cause of pathological alterations in various diseases, including diabetic retinopathy (1). Since normal retinal metabolism requires the consumption of a large amount of oxygen, hypoxia results in damage, or even death, of retinal neuronal cells, and other tissues and cells (2). Photoreceptor cells are the primary neurons of the visual pathway and damage to these cells severely affects visual functions.

Toll-like receptor 4 (TLR4) is a member of Toll-like receptors (TLRs) family, which are germline-encoded innate immune receptors that detect invading microbials and induce immune responses (3). TLR4 expression is upregulated via hypoxia-inducible factor- $1 \alpha(\mathrm{HIF}-1 \alpha)$ in response to hypoxia condition (4). Previous studies have shown that TLR4 is involved in retinal ischemic injury $(5,6)$. However, the role of TLR4 and its downstream NADPH oxidase 4 (NOX4) under hypoxic condition is not fully understood.

Progranulin is a multi-functional growth factor composed of 593 amino acids, which is expressed in various tissues in the body (7). Progranulin gene mutations are not only associated with numerous neurodegenerative diseases (8), but also serve regulatory roles in inflammatory diseases. It has been reported that progranulin suppressed TLR4-driven inflammatory response in osteoarthritic tissues (9). However, few studies have investigated the functions of progranulin in retinopathy. In order to determine the function of progranulin on the retina under hypoxic conditions, a hypoxia model was established using the classic method of intravitreal injection with cobalt chloride $\left(\mathrm{CoCl}_{2}\right)$. Exogenous progranulin was applied, after which, alterations in the function and morphology of mouse photoreceptors, and in retinal vascular leukostasis, were detected.

\section{Materials and methods}

Reagents and instruments. Progranulin, $\mathrm{CoCl}_{2}$, ketamine, xylazine and resatorvid were purchased from Sigma-Aldrich 
(Merck KGaA, Darmstadt, Germany). Peanut agglutinin (PNA; cat. no. FL-1071), goat anti-rabbit fluorescent secondary antibody (cat. no. FI-1000) and DAPI were purchased from Vector Laboratories, Inc. (Burlingame, CA, USA). The rabbit anti-mouse caspase-3 (cat. no. MAB835-SP), goat anti-mouse p53 (cat. no. AF1355-SP) and goat anti-mouse $\beta$-catenin (cat. no. AF1329-SP) primary antibodies were purchased from R\&D Systems, Inc. (Minneapolis, MN, USA). The rabbit anti-mouse tumor necrosis factor (TNF)- $\alpha$ (cat. no. sc-52746), nuclear factor (NF)- $\kappa$ B/p65 (cat. no. sc-8008), vascular endothelial growth factor (VEGF; cat. no. sc-7269), HIF-1 $\alpha$ (cat. no. sc-13515) and $\beta$-actin (cat. no. sc-517582) primary antibodies were purchased from Santa Cruz Biotechnology Inc. (Dallas, TX, USA). The rabbit anti-mouse s-opsin (cat. no. ABN1660) and NOX4 (cat. no. ABC271) primary antibodies were purchased from EMD Millipore (Billerica, MA, USA). The rabbit anti-mouse glycogen synthase kinase (GSK)-3 $\beta$ (cat. no. ab32391), TLR4 (cat. no. ab13867) primary antibodies, HRP conjugated goat anti-rabbit (cat. no. ab6721) and rabbit anti-goat (cat. no. ab6741) secondary antibodies were purchased from Abcam (Cambridge, UK). PBS, citric acid buffer and goat serum were purchased from Tiangen Biotech Co., Ltd. (Beijing, China). The western blotting electrophoresis system and the protein concentration determination reagent kit were purchased from Bio-Rad Laboratories, Inc. (Hercules, CA, USA). Sodium hyaluronate gel was purchased from Shandong Bausch \& Lomb Freda Pharmaceutical Co., Ltd. (Shandong, China). Compound tropicamide eye drops were purchased from Santen Pharmaceutical Co., Ltd. (Osaka, Japan). The micro-injectors were purchased from the Hamilton Company (Reno, NV, USA). The Espion electroretinography system was purchased from Diagnosys Systems, Inc. (Westford, MA, USA). The inverted fluorescence microscope was purchased from Olympus Corporation (Tokyo, Japan), and the spectrophotometer was purchased from SPECTRO Analytical Instruments GmbH (Kleve, Germany).

Experimental animals and groups. The study complied with the Animal Research: Reporting of In Vivo Experiments guidelines (10) and was conducted in strict accordance with the recommendations in the Guide for the Care and Use of Laboratory Animals of the National Institutes of Health (Bethesda, MD, USA). The present study was approved by the Committee on the Ethics of Animal Experiments, Nanchang University (Nanchang, China). A total of 48 healthy, inbred, male C57BL/6 mice (age, 8 weeks; weight, 20-30 g), without eye diseases, were purchased from the Department of Animal Sciences, Nanchang University. They were housed at a temperature of $18-23^{\circ} \mathrm{C}$, humidity of $40-60 \%$ with a 14 -h light/10-h dark cycle and food and water accessible at all times All experimental manipulations followed the regulations of the Institutional Animal Care and Use Committee, Nanchang University. The mice were numbered using ear tags and were randomly divided into two groups: Normal condition and hypoxic condition groups ( $\mathrm{n}=24 \mathrm{mice} / \mathrm{group})$.

Intravitreal injection with $\mathrm{CoCl}_{2}$ to establish a hypoxic model. The mice in the two groups were anesthetized with a mixture of ketamine $(100 \mathrm{mg} / \mathrm{kg})$ and xylazine $(10 \mathrm{mg} / \mathrm{kg})$ via intraperitoneal injection, and iodophor was used to disinfect the area around the eyes. A Hamilton micro-injector needle was inserted $1 \mathrm{~mm}$ outside the limbus in the optic nerve direction under a microscope. The drugs were slowly dispensed when the needle tip was observed to be in the pupillary area. In the hypoxic condition group, $2 \mu 19 \mathrm{mM} \mathrm{CoCl}_{2}$ was intravitreally injected into both eyes. In the normal condition group, $2 \mu \mathrm{l}$ PBS was intravitreally injected into both eyes. After $6 \mathrm{~h}$, 18 mice from each group received intraperitoneal anesthesia as above. The aforementioned intravitreal injection steps were repeated through the previous puncture sites. The right eyes of the mice in the two groups were injected with $0.5 \mu 110 \mathrm{mM}$ progranulin, and the left eyes were injected with an equal amount of PBS. The remaining 6 mice in each group were injected with $0.5 \mu 18 \mathrm{mM}$ resatorvid in both eyes; after $1 \mathrm{~h}$, the right eyes of these mice were injected with $0.5 \mu 110 \mathrm{mM}$ progranulin, and the left eyes with an equal amount of PBS.

Immunoblotting. Anesthetized mice were sacrificed by an experienced technician, 24 eyeballs from 12 mice were removed, and the retinal tissues were collected and placed in an Eppendorf tube containing $200 \mu \mathrm{l}$ RIPA lysis buffer (1\% Nonidet P-40 (NP-40), $1 \%$ sodium deoxycholate, $0.1 \%$ SDS, $0.15 \mathrm{M} \mathrm{NaCl}, 0.01 \mathrm{M}$ sodium phosphate, $\mathrm{pH}$ 7.2, 2 mM EDTA, $50 \mathrm{mM}$ sodium fluoride, $0.2 \mathrm{mM}$ fresh sodium orthovanadate and $100 \mathrm{U} / \mathrm{ml}$ protease inhibitor aprotinin). The samples were sonicated (100-200 W) for $10 \mathrm{sec}$ three times and were then centrifuged $(10,000 \mathrm{xg})$ for $20 \mathrm{~min}$ at $4^{\circ} \mathrm{C}$. The supernatant was collected, and the protein concentrations of the samples were determined. Equal amounts (50 $\mu \mathrm{g}$ /lane) of protein samples were boiled in loading buffer and subjected to SDS-PAGE (5\% stacking gel and $10 \%$ separating gel). The proteins were transferred onto polyvinylidene difluoride (PVDF) membrane for $120 \mathrm{~min}$. The membrane was blocked with $5 \%$ non-fat dried milk in TBST at $37^{\circ} \mathrm{C}$ for $2 \mathrm{~h}$ then incubated with the primary antibodies $(1: 1,000)$ at $4^{\circ} \mathrm{C}$ overnight. The following day, the membrane was washed in TBST (Tris-buffered saline with $0.1 \%$ Tween-20) three times and incubated with a horseradish peroxidase-conjugated secondary antibody $(1: 2,000)$ at room temperature for $1 \mathrm{~h}$. Pierce ECL Western Blotting Substrate (Thermo Fisher Scientific, Inc., Waltham, MA, USA) was added in a dark room for gel image analysis and measurement of the gray density values of protein bands using UVP GelDoc-It Imager (UVP, LLC, Phoenix, AZ, USA) and VisionWorks ${ }^{\circledR}$ Image Acquisition \& Analysis Software (version 7.0; UVP, LLC).

Electroretinogram (ERG). Following intravitreal injection of progranulin or PBS, mydriasis was induced in 12 mice from each group using compound tropicamide eye drops; subsequently, the mice were placed in a dark room overnight. The next day, following administration of intraperitoneal anesthesia with mixture of ketamine $(100 \mathrm{mg} / \mathrm{kg})$ and xylazine $(10 \mathrm{mg} / \mathrm{kg})$, the mice were placed onto a heating pad. Reference and grounding electrodes were inserted into the palate and tail. A platinum corneal electrode was placed at the corneas of both eyes and lubricated using sodium hyaluronate gel. These manipulations were all performed in a dark room with dim red-light illumination. The light intensities $(0.0004$, $0.04,4,400$ and $2,000 \mathrm{~cd} . \mathrm{s} / \mathrm{m}^{2}$ ) were established in the ERG to record the scotopic ERG reaction. The light was turned on for 

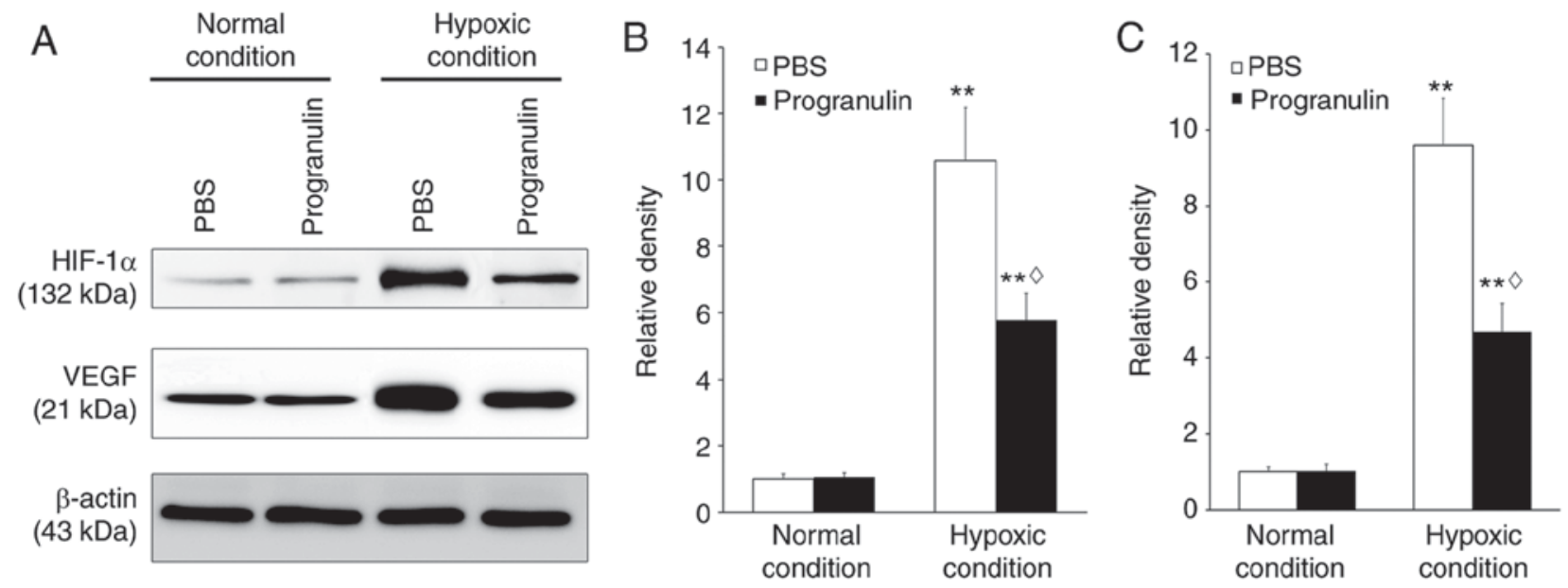

Figure 1. Detection of the retinal expression levels of HIF-1 $\alpha$ and VEGF. (A) Protein expression levels were detected using western blotting. Statistical analysis of (B) HIF-1 $\alpha$ and (C) VEGF expression. ${ }^{* *} \mathrm{P}<0.01$ vs. the normal condition; ${ }^{\circ} \mathrm{P}<0.05$ vs. the PBS group. Data are presented as the means \pm standard deviation, $\mathrm{n}=6$. HIF, hypoxia-inducible factor; VEGF, vascular endothelial growth factor.

adaption for $10 \mathrm{~min}$ and the photopic ERG reaction was then recorded under $2,000 \mathrm{~cd} . \mathrm{s} / \mathrm{m}^{2}$ light intensity.

Immunofluorescence co-localization. Following completion of the ERG, six anesthetized mice from each group were sacrificed under anesthetic by a well-trained technician. The eyeballs were removed and directly placed in $4 \%$ paraformaldehyde for $1 \mathrm{~h}$ of fixation at room temperature. Following the removal of the cornea and lens, the eyeballs were placed in $4 \%$ paraformaldehyde again for fixation for $15 \mathrm{~min}$ at room temperature. After washing in PBS and dehydrating in gradient alcohol, the eyeballs were cleared in xylene I and xylene II, immersed in paraffin, embedded, sectioned $(5-\mu \mathrm{m})$ and the slides dried for $30 \mathrm{~min}$ on a slide warmer at $37^{\circ} \mathrm{C}$. The paraffin-embedded sections were deparaffinized in xylene I and xylene II, rinsed in anhydrous alcohol and placed in gradient alcohol. Antigen retrieval was performed using $0.01 \mathrm{~mol} / 1$ citric acid buffer at $98^{\circ} \mathrm{C}$ for $20 \mathrm{~min}$. The sections were allowed to cool, rinsed three times with PBS, incubated with goat serum in a moisture box for $1 \mathrm{~h}$ and were then incubated with primary antibodies as above $(1: 1,000)$ at $4^{\circ} \mathrm{C}$ overnight. The primary antibody was replaced with $\mathrm{PBS}$ in the negative control group. Subsequently, the sections were rinsed three times with PBS and were incubated with a secondary antibody as above $(1: 2,000)$ and PNA at room temperature for $1 \mathrm{~h}$. Finally, the sections were mounted in DAPI containing an anti-quenching agent. The results were observed under a fluorescence microscope.

Retinal vascular leukostasis assay. A total of 6 mice from each group were randomly selected following completion of the ERG. The mice were intraperitoneally anesthetized with a mixture of ketamine $(100 \mathrm{mg} / \mathrm{kg})$ and xylazine (10 mg/kg) (Sigma-Aldrich; Merck KGaA), after which, the thoracic skin and ribs of the mice were cut to expose the thoracic cavity. The descending aorta was clamped, the right atrial appendage was cut, and a $27 \mathrm{G}$ needle was inserted into the left ventricle. Non-adherent leukocytes were washed away by perfusion with a 10-ml mixture of PBS and heparin $(0.1 \mathrm{mg} / \mathrm{ml})$. Subsequently, the adherent leukocytes were labeled with a mixed solution containing $20 \mu \mathrm{g} / \mathrm{ml}$ PBS and fluorescein isothiocyanate (FITC)-labeled concanavalin A (ConA; 5 mg/kg; Sigma-Aldrich; Merck KGaA). FITC-ConA that did not interact with the leukocytes was washed away using $10 \mathrm{ml}$ PBS. Following sacrifice of the anesthetized mice via spinal dislocation by a well-trained technician, six pairs of eyeballs from each group were removed and directly placed into $4 \%$ paraformaldehyde for fixation for $1 \mathrm{~h}$ at room temperature. Finally, the retinal tissues were flat mounted, and leukocytes were counted under a fluorescence microscope.

Statistical analysis. In vivo experiments were repeated $\geq 3$ times. The statistical analyses were performed using SPSS version 17.0 (SPSS, Inc., Chicago, IL, USA). The gray density values of the western blots and the numbers of adherent leukocytes were expressed as means \pm standard deviation. The ERG results were expressed as means \pm standard error of the mean. The comparisons between the parameters in the differently treated eyes were performed using one-way analysis of variance followed by a post hoc Student-Newman-Keul test. $\mathrm{P}<0.05$ was considered to indicate a statistically significant difference.

\section{Results}

Establishment of the hypoxic mouse model. Retinal HIF-1 $\alpha$ and VEGF expression were detected to verify whether hypoxia was successfully induced. Immunoblotting revealed that the expression levels of HIF-1 $\alpha$ were significantly upregulated under hypoxic conditions compared with under normal conditions $(\mathrm{P}<0.01)$. However, under hypoxic conditions, the expression of HIF-1 $\alpha$ in the progranulin-treated eyes was $\sim 54.58 \%$ that in the PBS-treated eyes ( $<<0.05$; Fig. $1 \mathrm{~A}$ and $\mathrm{B})$. Similar results were obtained regarding the expression levels of VEGF, which were also significantly upregulated under hypoxic conditions compared with under normal conditions $(\mathrm{P}<0.01)$. However, under hypoxic conditions, the expression of retinal VEGF in the progranulin-treated eyes was $\sim 48.91 \%$ that in the PBS-treated eyes $(\mathrm{P}<0.05$; Fig. $1 \mathrm{~A}$ and $\mathrm{C})$.

Progranulin attenuates the functional damage of photoreceptor cells during hypoxia. Under hypoxic conditions, 

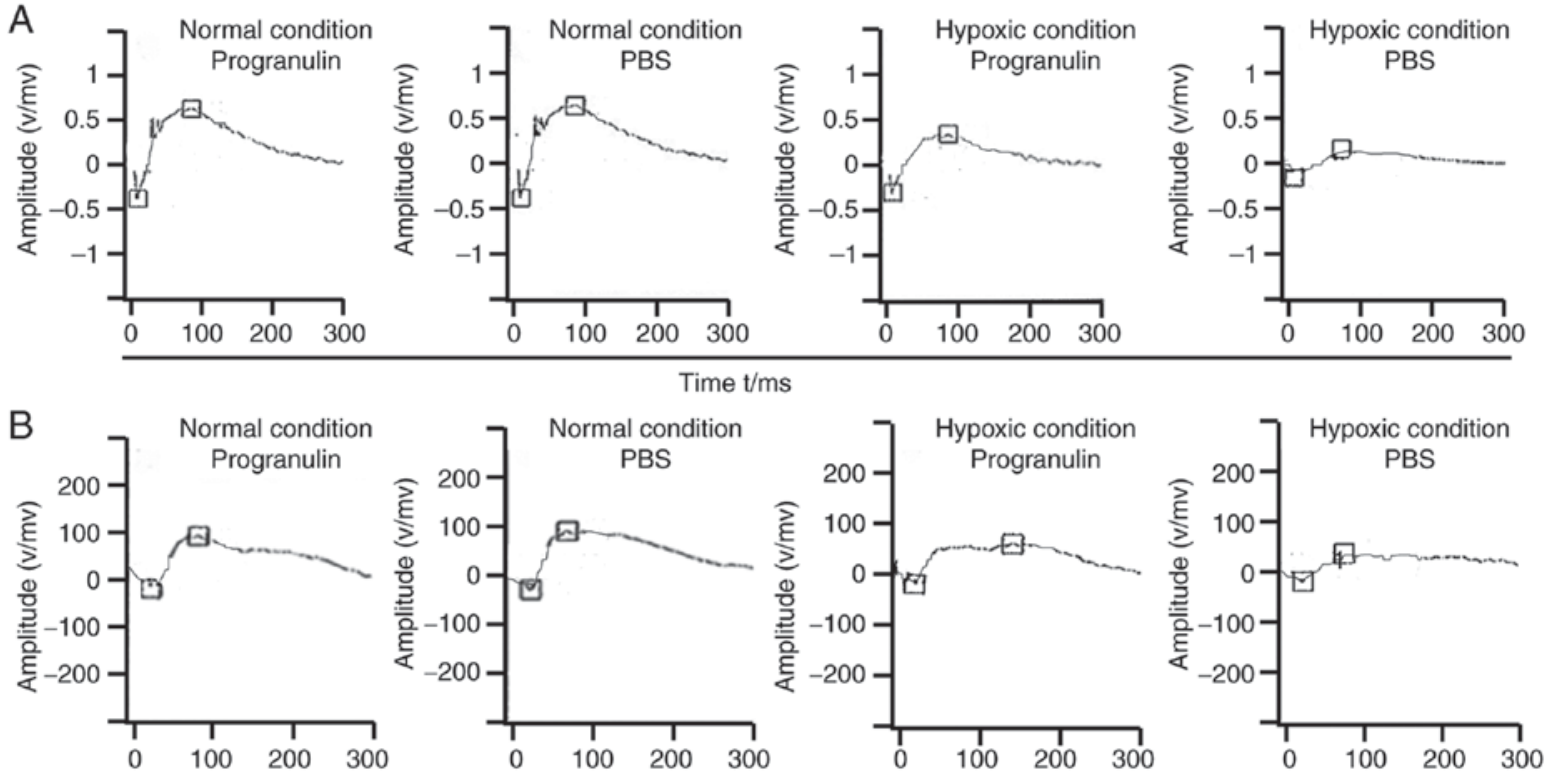

Time $t / m s$

C
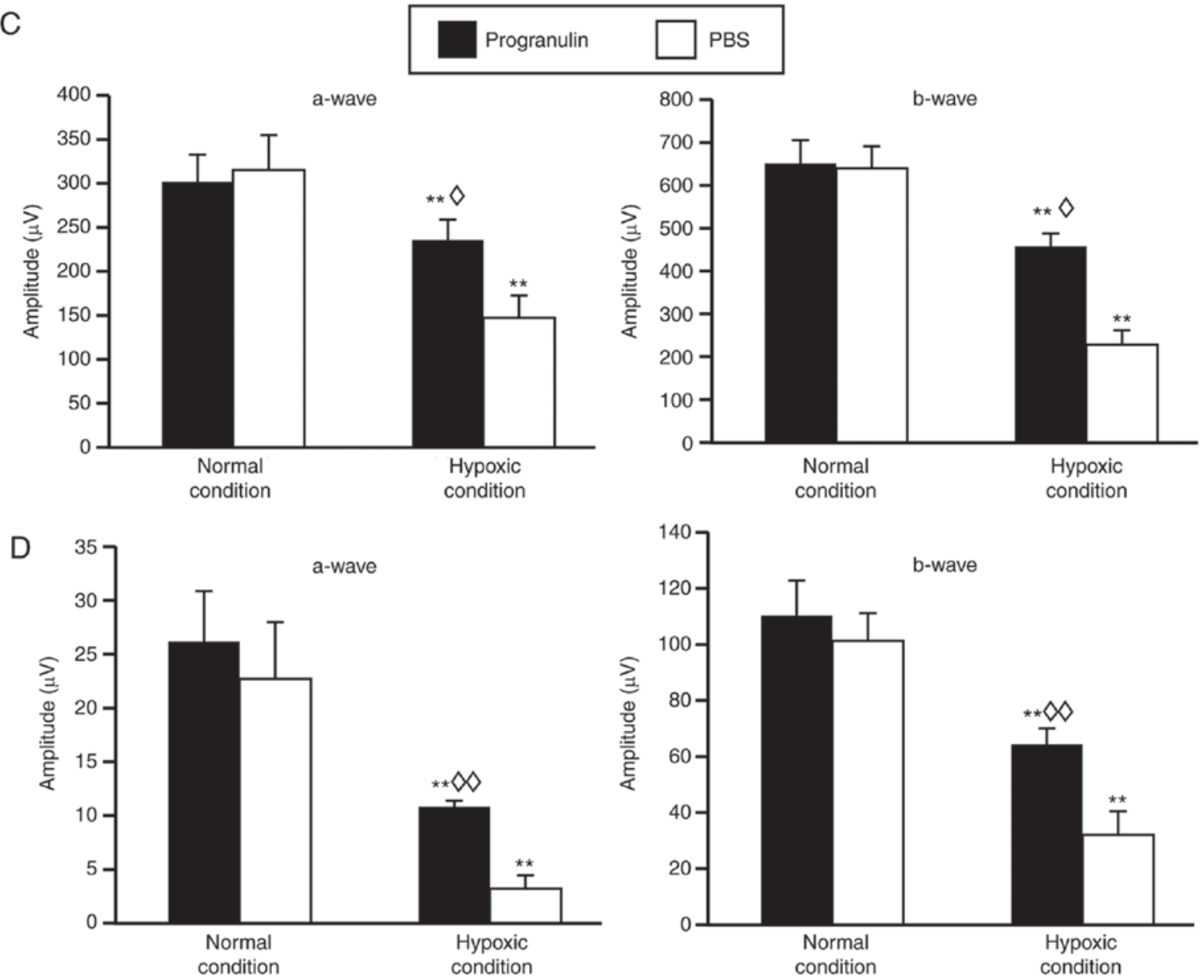

Figure 2. ERGs of mice following intravitreal injection of exogenous progranulin under hypoxic conditions. Typical waveforms of the (A) scotopic and (B) photopic ERGs under normal and hypoxic conditions. Statistical analysis of the (C) scotopic ERG and (D) photopic ERG. **P $<0.01$ vs. the normal condition; ${ }^{\circ} \mathrm{P}<0.05,{ }^{\diamond} \mathrm{P}<0.01$ vs. the PBS group. Data are presented as means \pm standard error of the mean, $\mathrm{n}=12$. ERG, electroretinogram; OD, oculus dexter, the right eye; OS, oculus sinister, the left eye.

the amplitudes of a-waves and b-waves in the mouse scotopic ERG (Fig. 2A) and photopic ERG (Fig. 2B) were reduced compared with in the normal control group, and statistical analysis demonstrated that the differences were significant (Fig. 2C and D). In addition, the ERG amplitudes were reduced in the PBS-injected eyes compared 


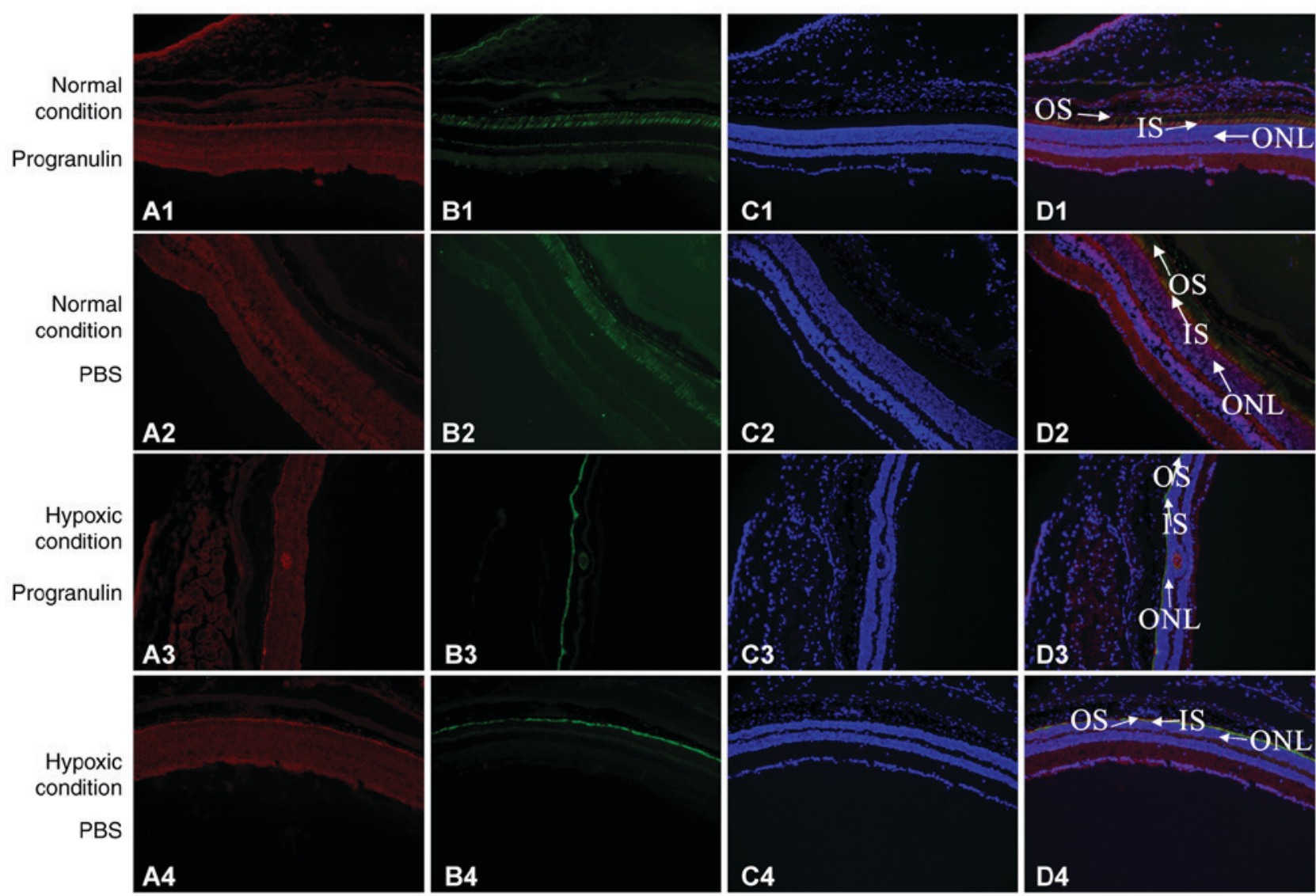

Figure 3. Detection of morphological alterations in mouse retinal photoreceptors under the hypoxic condition using immunofluorescence co-localization. Group 1, normal condition + progranulin injection; Group 2, normal condition + PBS injection; Group 3, hypoxic condition + progranulin injection; Group 4, hypoxic condition + PBS injection. (A) Red, s-opsin. (B) Green, PNA. (C) Blue, DAPI. (D) Merge. Original magnification, x100; IS, inner segment of cone cells; ONL, outer nuclear layer; OS, outer segment of cone cell.

with in the progranulin-injected eyes (Fig. 2A and B), and statistical analysis demonstrated that the differences were significant (Fig. 2C and D).

Progranulin attenuates morphological damage of photoreceptor cells during hypoxia. Immunofluorescence co-localization was performed to detect the morphology of retinal photoreceptor cells. S-opsin was used to label the outer segments of the cone cells, PNA was used to label the whole cone cells and DAPI was used to label the nuclei. S-opsin and PNA reflected morphological alterations to the rod cells, as did the thickness of the outer nuclear layer. The results demonstrated that the thickness of the retinal outer nuclear layer was significantly decreased in mice under hypoxic conditions compared with in the normal control group. Furthermore, the thickness of the retinal outer nuclear layer was smaller in the PBS-injected eyes compared with in the progranulin-injected eyes. The morphology of the cone cells also exhibited significant alterations compared with the normal control group, with the morphology of the outer segments becoming shorter, smaller and disordered. The changes were more evident in the cone cells in the PBS-injected eyes than in the progranulin-injected eyes, and the cone cells were sparser in the former group (Fig. 3).

Progranulin reduces the number of adherent leukocytes in the retinal blood vessels under hypoxic conditions. Adherent leukocytes in mouse retinal blood vessels are an indicator used to measure retinal inflammatory responses (11). The number of adherent leukocytes under hypoxic conditions was significantly increased compared with in the normal control group (Fig. 4). In addition, the number of adherent leukocytes in the progranulin-injected eyes (Fig. 4A and C) was $\sim 52.31 \%$ that in the PBS-injected eyes (Fig. 4B and D); these differences were significant (Fig. 4E).

Progranulin exerts retinal protective effects through regulation and inhibition of the TLR4-NOX4 signaling pathway. The expression levels of caspase signaling pathway-associated proteins, caspase- 3 and $\mathrm{p} 53$, and $\mathrm{Wnt} / \beta$-catenin signaling pathway-associated proteins, GSK-3 $\beta$ and $\beta$-catenin, were evaluated in the retina. The expression levels of caspase- 3 , p53, GSK-3 $\beta$ and $\beta$-catenin were significantly upregulated under hypoxic conditions compared with in the normal control group; however, the differences in expression between the progranulin- and PBS-injected eyes were not significant (Fig. 5A-D). Therefore, it was concluded that progranulin did not exert its function through the regulation of these two signaling pathways.

The expression levels of TLR4-NOX4 signaling pathwayassociated proteins, TLR4 and NOX4, and downstream inflammatory factors, TNF- $\alpha$ and NF- $\kappa B$, were also examined in the retina. The expression levels of TLR4, NOX4, TNF- $\alpha$ and NF- $\mathrm{KB}$ were significantly upregulated under hypoxic 

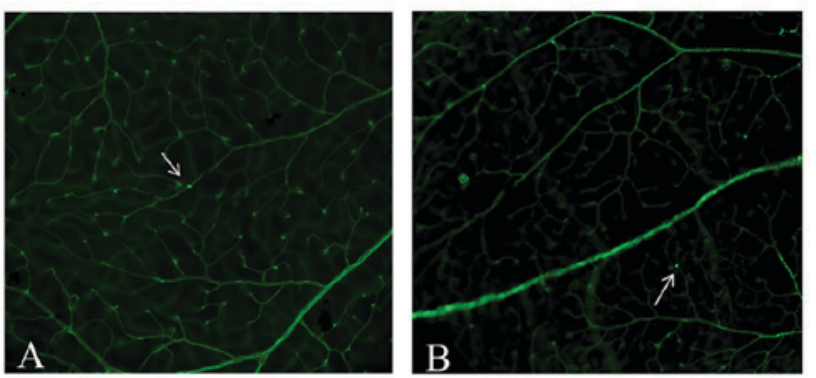

E
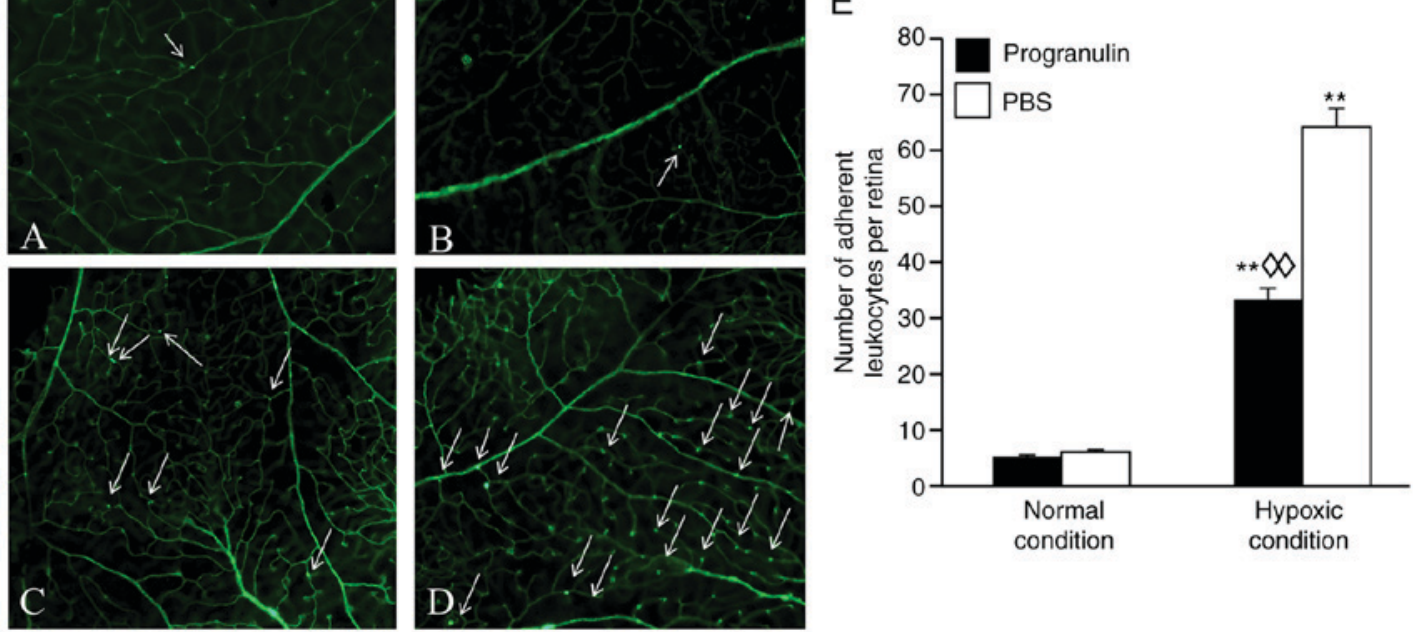

Figure 4. Number of adherent leukocytes in retinal blood vessels under hypoxic conditions, as determined using fluorescein isothiocyanate-conjugated concanavalin A staining. (A) Normal condition + progranulin injection group. (B) Normal condition + PBS injection group. (C) Hypoxic condition + progranulin injection group. (D) Hypoxic condition + PBS injection group. White arrows indicate adherent leukocytes. (E) Statistical analysis. Original magnification, x800. Data are presented as the means \pm standard deviation, $n=6 .{ }^{* *} \mathrm{P}<0.01$ vs. the normal condition; ${ }^{\triangleright \triangleright} \mathrm{P}<0.01$ vs. the PBS group.

conditions compared with under normal conditions. These results suggested that the TLR4-NOX4 signaling pathway was activated in the retina under hypoxic conditions. In addition, the expression levels of the aforementioned proteins were significantly lower in the progranulin-injected eyes compared with in the PBS-injected eyes (Fig. 5E and F). Subsequently, resatorvid, a TLR4 inhibitor originally developed to stop the progression of sepsis (12), was intravitreally injected to observe its effects on the mouse retina. Western blotting results demonstrated that the expression levels of TLR4 and NOX4, and the downstream proteins, TNF- $\alpha$ and NF- $\kappa B$, were not significantly different between PBS- and progranulin-injected eyes (Fig. 4G and $\mathrm{H}$ ). These results preliminarily suggested that progranulin may exert its function through regulation and inhibition of the TLR4-NOX4 signaling pathway.

\section{Discussion}

Progranulin is a multi-functional growth factor (13), which serves an important role in various pathophysiological processes, including tumor development, inflammatory responses, nerve cell survival and axonal growth regulation. In addition, the progranulin gene affects the axonal growth of neurons through regulation of GSK $3 \beta$, which is an important component of the $\mathrm{Wnt} / \beta$-catenin signaling pathway. Progranulin gene knockout in nervous system cells has been reported to increase the expression of $\mathrm{Wnt} / \beta$-catenin signaling pathway-associated proteins (14). Progranulin also inhibits the caspase pathway; activation of caspase signaling pathway-associated proteins in the cortex is intensified in progranulin gene knockout mice, and cortical neuronal apoptosis is increased (15). Progranulin is also an immune regulatory factor that serves a regulatory role in inflammatory diseases. Local injection of progranulin into mice in a multiple arthritis model inhibits the neutrophil-induced inflammatory response (16). Tang et al (17) demonstrated that progranulin is a novel ligand for the TNF receptor that interacts with its extracellular domain; progranulin blocks TNF- $\alpha$-mediated signaling pathways, including NF- $\kappa \mathrm{B}$, by competing with TNF- $\alpha$ for its receptors and thus serves important anti-inflammatory roles in rheumatoid arthritis. Therefore, progranulin is considered a protein with extensive functionality. However, to date, few reports have studied its function in retinopathy. Tsuruma et al (18) reported that progranulin serves a protective role in a light-induced retina degeneration model, which is used to mimic age-related macular degeneration (19). To the best of our knowledge, the role of progranulin under hypoxic conditions has not yet been reported, and requires further investigation.

Hypoxia is involved in various retinopathies, including diabetic retinopathy (20), central retinal vein occlusion and macular edema (21). Investigations into the pathological alterations of retinal tissues under hypoxic conditions may contribute to understanding the pathogenic mechanisms underlying these diseases. The chemical drug most commonly used to simulate the hypoxic environment is $\mathrm{CoCl}_{2}$. The principle underlying this method is to block intracellular respiratory chain transfer and cause chemical hypoxia in cells $(22,23)$. Intravitreal injection of $\mathrm{CoCl}_{2}$, a chemical hypoxia-mimicking agent, is an emerging in vivo method to simulate retinal tissue hypoxia in rodents (23). This process has a short cycle for the establishment of a hypoxia model and is convenient to use. A previous study performed intravitreal injection of various $\mathrm{CoCl}_{2}$ concentrations in rats, in order to investigate the optimal concentration required to induce hypoxia, and the results have demonstrated an optimal effect at $9 \mathrm{mM} \mathrm{CoCl}_{2}$ (23). Therefore, this concentration was adopted in the present study.

ERG is mainly used to detect the function of retinal photoreceptor cells. This method uses low light and a white standard flash to stimulate photoreceptors to produce electric potential, which can be recorded non-invasively using a 

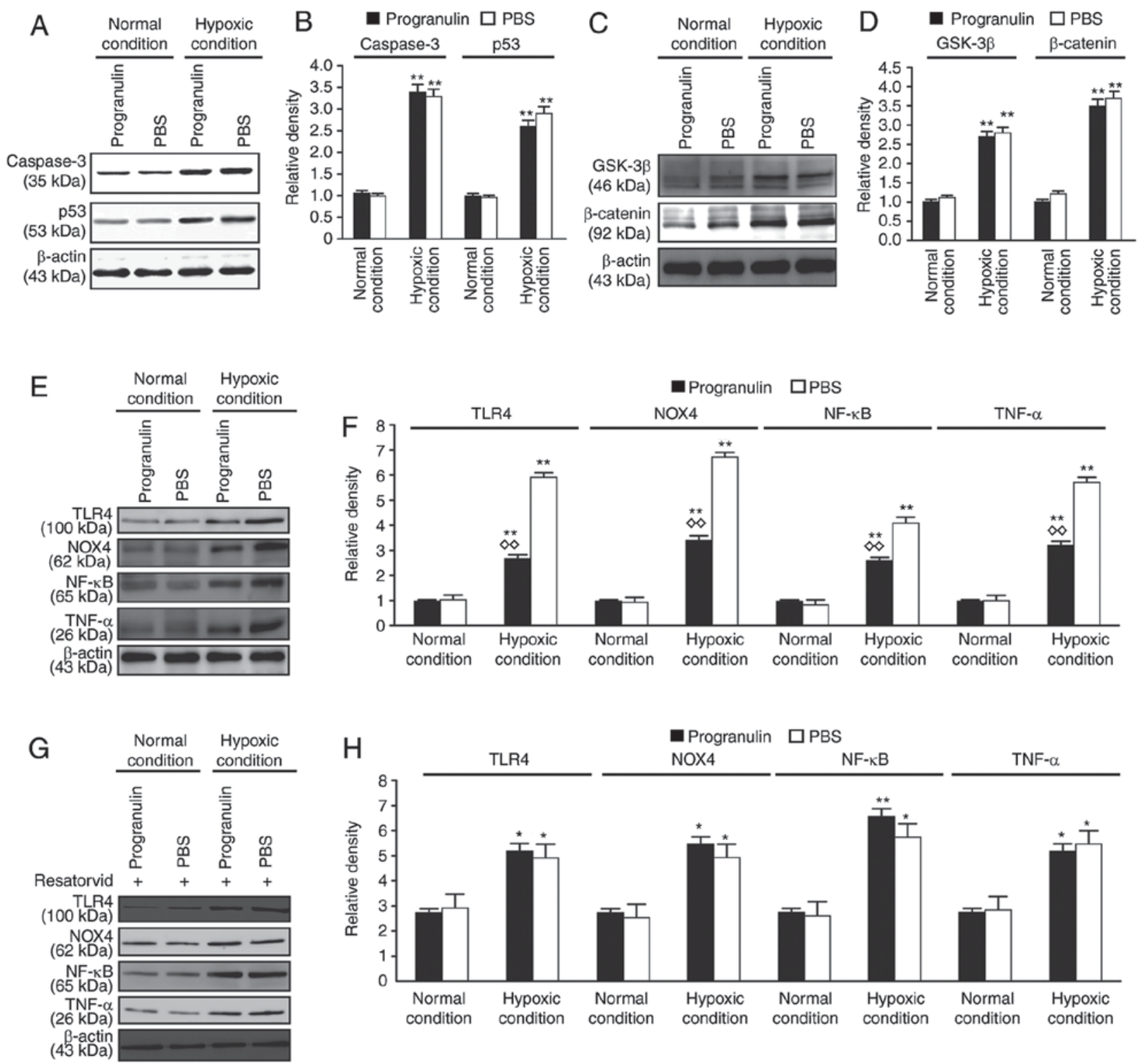

Figure 5. Detection of the expression levels of caspase, Wnt/ $\beta$-catenin and TLR4-NOX4 signaling pathway-associated proteins by western blotting. (A) Detection of the expression levels of the caspase signaling pathway-associated proteins, caspase-3 and p53, using western blotting. (B) Statistical analysis. (C) Detection of the expression levels of the Wnt/ $\beta$-catenin signaling pathway-associated proteins, GSK-3 $\beta$ and $\beta$-catenin, using western blotting. (D) Statistical analysis . (E) Detection of the expression levels of TLR4, NOX4, TNF- $\alpha$ and NF- $\mathrm{kB} / \mathrm{p} 65$ using western blotting. (F) Statistical analysis. (G) Detection of the expression levels of TLR4, NOX4, TNF- $\alpha$ and NF- $\mathrm{kB} / \mathrm{p} 65$ following treatment with the inhibitor resatorvid. (H) Statistical analysis. Data are presented as the means \pm standard error of the mean, $\mathrm{n}=6 .{ }^{*} \mathrm{P}<0.05,{ }^{* *} \mathrm{P}<0.01$ vs. the normal condition; ${ }^{\triangleright \bullet} \mathrm{P}<0.01$ vs. the PBS group. GSK- $3 \beta$, glycogen synthase kinase-3 $\beta$;

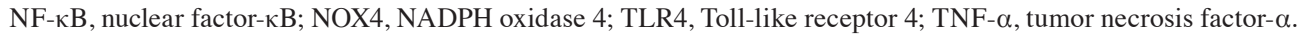

platinum electrode on the corneal surface (24). Scotopic ERG reflects the functional status of rod cells, and photopic ERG is typically used to reflect the functional status of cone cells. The present study demonstrated that although the amplitudes of a-waves and b-waves in the ERGs of mice in the hypoxic condition group were lower compared with the amplitudes in the normal condition group, the reduction in the amplitude was lower in progranulin-injected eyes compared with in PBS-injected eyes. These results suggested that progranulin may exert protective effects on the function of photoreceptor cells. Following the completion of the functional evaluation, immunofluorescence co-localization was performed using markers for whole cone cells (PNA) and the outer segment of cone cells (s-opsin) (25), in order to observe alterations in cone cell structure. Furthermore, DAPI staining was used to observe alterations in the structure of the outer nuclear layer formed by the rod cells. The number of rod cells was decreased in PBS-injected eyes compared with in progranulin-injected eyes, as revealed by a decrease in the thickness of the outer nuclear layer; in addition, the arrangement of cone cells was sparse, and the morphology of the outer segment was short and small. These results suggested that the occurrence of photoreceptor cell damage and death in the progranulin-injected eyes was relatively low, even though the photoreceptor cells experienced a considerable number of deaths under hypoxic conditions in the present study. These findings suggested that progranulin may exert protective effects on photoreceptor cells. 
The inflammatory response is an important step in microvascular diseases. Numerous studies have reported that, in diabetic animal models, the number of retinal leukocytes and their adhesive ability is increased, whereas deformability is decreased $(11,26)$. The passive deformability of leukocytes decreases when passing through capillaries with smaller diameters; therefore, the leukocyte adhesion rate increases. Additionally, the increase in leukocyte adhesion rate is more evident with the progression of disease; therefore, the retinal vascular leukostasis assay can be used to analyze the levels of the inflammatory response. The present study demonstrated that the number of adherent leukocytes was higher in progranulin-injected eyes under hypoxic conditions compared with in the normal condition group, but was only $52.31 \%$ that in PBS-injected eyes. As aforementioned, progranulin is associated with the $\mathrm{Wnt} / \beta$-catenin, caspase and TLR4 signaling pathways. Therefore, western blotting was performed to screen relevant signaling pathways. The results demonstrated that the protective effects of progranulin on the retina under hypoxic conditions did not occur through regulation of the caspase and Wnt/ $\beta$-catenin signaling pathways; instead, the regulation involved inhibition of the TLR4-NOX4 signaling pathway.

In conclusion, the present study reported that progranulin may exert protective effects on mouse photoreceptor functions and morphology under $\mathrm{CoCl}_{2}$-induced hypoxia. Additionally, progranulin reduced the number of adherent leukocytes in retinal blood vessels. These functions occurred through inhibition of the TLR4-NOX4 signaling pathway. The specific mechanism and function underlying regulation of the TLR4-NOX4 signaling pathway by progranulin are currently under investigation. These results suggested that inhibition of TLR4-NOX4 signaling by progranulin may become a new direction for future therapy of retinal hypoxia.

\section{Acknowledgements}

Not applicable.

\section{Funding}

The present study was supported by the National Natural Science Foundation of China (grant nos. 81460088 and 81760176), the Jiangxi Provincial Training Program for Distinguished Young Scholars (grant no. 20171BCB23092), the Jiangxi Provincial Key R\&D Program (grant no. 20171BBG70099), the Jiangxi Provincial Natural Science Foundation for Youth Scientific Research (grant no. 20171BAB215032) and the Youth Scientific Research Foundation of the Second Affiliated Hospital of Nanchang University (grant no. 2014YNQN12011).

\section{Availability of data and materials}

The datasets used and/or analyzed during the current study are available from the corresponding author on reasonable request.

\section{Authors' contributions}

ZPY and KS designed the study and performed the experiments. MJY performed the experiments. YLZ analyzed the data, and KS wrote the manuscript.

\section{Ethics approval and consent to participate}

The present study complied with the Animal Research: Reporting of in vivo Experiments guidelines and was conducted in strict accordance with the recommendations in the Guide for the Care and Use of Laboratory Animals of the National Institutes of Health. This study was approved by the Committee on the Ethics of Animal Experiments of Nanchang University.

\section{Patient consent for publication}

Not applicable.

\section{Competing interests}

The authors declare that they have no competing interests.

\section{References}

1. Cheng L, Yu H, Yan N, Lai K and Xiang M: Hypoxia-inducible factor-1 $\alpha$ target genes contribute to retinal neuroprotection. Front Cell Neurosci 11: 20, 2017.

2. Kaur C, Foulds WS and Ling EA: Hypoxia-ischemia and retinal ganglion cell damage. Clin Ophthalmol 2: 879-889, 2008.

3. Akira S, Uematsu S and Takeuchi O: Pathogen recognition and innate immunity. Cell 124: 783-801, 2006.

4. Kim SY, Choi YJ, Joung SM, Lee BH, Jung YS and Lee JY: Hypoxic stress up-regulates the expression of Toll-like receptor 4 in macrophages via hypoxia-inducible factor. Immunology 129: 516-524, 2010.

5. Dvoriantchikova G, Barakat DJ, Hernandez E, Shestopalov VI and Ivanov D: Toll-like receptor 4 contributes to retinal ischemia/reperfusion injury. Mol Vis 16: 1907-1912, 2010.

6. Liu L, Jiang Y and Steinle JJ: Toll-like receptor 4 reduces occludin and zonula occludens 1 to increase retinal permeability both in vitro and in vivo. J Vasc Res 54: 367-375, 2017.

7. Tolkatchev D, Malik S, Vinogradova A, Wang P, Chen Z, Xu P, Bennett HP, Bateman A and Ni F: Structure dissection of human progranulin identifies well-folded granulin/epithelin modules with unique functional activities. Protein Sci 17: 711-724, 2008.

8. Petkau TL and Leavitt BR: Progranulin in neurodegenerative disease. Trends Neurosci 37: 388-398, 2014.

9. Abella V, Scotece M, Conde J, López V, Pirozzi C, Pino J Gómez R, Lago F, González-Gay MÁ and Gualillo O: The novel adipokine progranulin counteracts IL-1 and TLR4-driven inflammatory response in human and murine chondrocytes via TNFR1. Sci Rep 6: 20356, 2016.

10. Drummond GB, Paterson DJ and McGrath JC: ARRIVE: New guidelines for reporting animal research. J Physiol 588: 2517, 2010

11. Schröder S, Palinski W and Schmid-Schönbein GW: Activated monocytes and granulocytes, capillary nonperfusion, and neovascularization in diabetic retinopathy. Am J Pathol 139: 81-100, 1991.

12. Sha T, Sunamoto M, Kitazaki T, Sato J, Ii M and Iizawa Y: Therapeutic effects of TAK-242, a novel selective Toll-like receptor 4 signal transduction inhibitor, in mouse endotoxin shock model. Eur J Pharmacol 571: 231-239, 2007.

13. Jian J, Li G, Hettinghouse A and Liu C: Progranulin: A key player in autoimmune diseases. Cytokine 101: 48-55, 2018.

14. Rosen EY, Wexler EM, Versano R, Coppola G, Gao F, Winden KD, Oldham MC, Martens LH, Zhou P, Farese RV Jr and Geschwind DH: Functional genomic analyses identify pathways dysregulated by progranulin deficiency, implicating Wnt signaling. Neuron 71: 1030-1042, 2011.

15. Kumar-Singh S: Progranulin and TDP-43: Mechanistic links and future directions. J Mol Neurosci 45: 561-573, 2011.

16. Jian J, Zhao S, Tian Q, Gonzalez-Gugel E, Mundra JJ, Uddin SM, Liu B, Richbourgh B, Brunetti R and Liu CJ: Progranulin directly binds to the CRD2 and CRD3 of TNFR extracellular domains. FEBS Lett 587: 3428-3436, 2013.

17. Tang W, Lu Y, Tian QY, Zhang Y, Guo FJ, Liu GY, Syed NM, Lai Y, Lin EA, Kong L, et al: The growth factor progranulin binds to TNF receptors and is therapeutic against inflammatory arthritis in mice. Science 332: 478-484, 2011. 
18. Tsuruma K, Yamauchi M, Sugitani S, Otsuka T, Ohno Y, Nagahara Y, Ikegame Y, Shimazawa M, Yoshimura S, Iwama T and Hara H: Progranulin, a major secreted protein of mouse adipose-derived stem cells, inhibits light-induced retinal degeneration. Stem Cells Transl Med 3: 42-53, 2014.

19. Enoki M, Shinto S, Matsuoka Y, Otsuka A, Kaidzu S, Tanito M, Shibata T, Uchida K, Ohira A, Yamato M and Yamada KI: Lipid radicals cause light-induced retinal degeneration. Chem Commun (Camb) 53: 10922-10925, 2017.

20. Arden GB and Sivaprasad S: Hypoxia and oxidative stress in the causation of diabetic retinopathy. Curr Diabetes Rev 7: 291-304, 2011

21. Stephen J Ryan (ed): Retina. Vol 1. 4th edition. Elsevier, Singapore, 2010

22. Niwa M, Aoki H, Hirata A, Tomita H, Green PG and Hara A: Retinal cell degeneration in animal models. Int J Mol Sci 17: E110, 2016.

23. Hara A, Niwa M, Aoki H, Kumada M, Kunisada T, Oyama T, Yamamoto T, Kozawa O and Mori H: A new model of retinal photoreceptor cell degeneration induced by a chemical hypoxia-mimicking agent, cobalt chloride. Brain Res 1109: 192-200, 2006.
24. Abcouwer SF and Gardner TW: Diabetic retinopathy: Loss of neuroretinal adaptation to the diabetic metabolic environment. Ann NY Acad Sci 1311: 174-190, 2014.

25. Gaillard F, Kuny S and Sauvé Y: Topographic arrangement of $\mathrm{S}$-cone photoreceptors in the retina of the diurnal Nile grass rat (Arvicanthis niloticus). Invest Ophthalmol Vis Sci 50: 5426-5434, 2009.

26. Aiello LP, Avery RL, Arrigg PG, Keyt BA, Jampel HD, Shah ST, Pasquale LR, Thieme H, Iwamoto MA, Park JE, et al: Vascular endothelial growth factor in ocular fluid of patients with diabetic retinopathy and other retinal disorders. N Engl J Med 331: 1480-1487, 1994

This work is licensed under a Creative Commons Attribution-NonCommercial-NoDerivatives 4.0 International (CC BY-NC-ND 4.0) License. 